\title{
Repulsion and Attraction of Axons by Semaphorin3D Are Mediated by Different Neuropilins In Vivo
}

\author{
Marc A. Wolman, ${ }^{1,2}$ Yan Liu, ${ }^{1}$ Hiroshi Tawarayama, ${ }^{3}$ Wataru Shoji, ${ }^{3}$ and Mary C. Halloran ${ }^{1,2}$ \\ ${ }^{1}$ Departments of Zoology and Anatomy and ${ }^{2}$ Neuroscience Training Program, University of Wisconsin, Madison, Wisconsin 53706, and ${ }^{3}$ Institute of \\ Development, Aging, and Cancer, Tohoku University, Sendai 980-8575, Japan
}

\begin{abstract}
Class 3 semaphorins are known to repel and/or sometimes attract axons; however, their role in guiding developing axons in the CNS in vivo is still essentially unknown. We investigated the role of Semaphorin3D (Sema3D) in the formation of the early axon pathways in the zebrafish CNS. Morpholino knock-down shows that Sema3D is essential for the correct formation of two early axon pathways. Sema3D appears to guide axons of the nucleus of the medial longitudinal fasciculus (nucMLF) by repulsion and modulation of fasciculation. In contrast, Sema3D appears to be attractive to telencephalic neurons that form the anterior commissure (AC). Knock-down of Neuropilin-1A (Npn-1A) phenocopied the effects of Sema3D knock-down on the nucMLF axons, and knock-down of either Npn-1A or Npn-2B phenocopied the defects of the AC. Furthermore, simultaneous partial knock-down experiments demonstrated genetic interactions among Sema3D, Npn-1A, and Npn-2B. Together, these data support the hypothesis that Sema3D may act as a repellent through receptors containing Npn-1A and as an attractant via receptors containing Npn-1A and Npn-2B.
\end{abstract}

Key words: semaphorin; neuropilin; axon guidance; fasciculation; chemoattraction; chemorepulsion

\section{Introduction}

During the development of the complex connections of the CNS, axons interpret multiple molecular signals provided by surrounding cells to navigate to their targets. Semaphorins are one family of molecules that can direct growing axons. Individual class 3-secreted semaphorins (Sema3) can function as chemorepellants or chemoattractants and sometimes both, depending on the particular axon type examined (Fiore and Puschel, 2003; Fujisawa, 2004). The receptor complex for class 3 semaphorins consists of a neuropilin ligand-binding component, neuropilin-1 (Npn-1) or neuropilin-2 (Npn-2), and a plexin signaltransducing element (Tamagnone and Comoglio, 2000). Binding assays indicate that all class 3 semaphorins tested bind Npn-1 with varying affinities (Feiner et al., 1997; He and TessierLavigne, 1997; Kolodkin and Ginty, 1997; Takahashi et al., 1998). Sema3B, -3C, and -3F have been shown to bind Npn-2, whereas Sema3A does not (Chen et al., 1997; Xu et al., 1998); however, semaphorins with similar Npn binding affinities in vitro bind to different cellular sites in situ (Feiner et al., 1997). Moreover, Sema3B and Sema3C, which bind with equal affinity to Npn-1 or Npn-2, act as antagonists at Npn-1 receptors and agonists at Npn-2 receptors (Chen et al., 1998; Takahashi et al., 1998). These

Received June 15, 2004; revised Aug. 16, 2004; accepted Aug. 16, 2004.

This work was supported by National Science Foundation (NSF) Grant IBN-0110654 and National Institute of Neurological Disorders and Stroke Grant NS42228 to M.C.H. The NSF supported acquisition of the confocal microscope (Grant NSF9724515 to Jim Pawley, Department of Zoology, University of Wisconsin). We thank Kate Kalil for critical reading of this manuscript and Aidan Reilly for fish care.

Correspondence should be addressed to Mary C. Halloran, Department of Zoology, 1117 West Johnson Street, Madison, WI 53706.E-mail: mchalloran@wisc.edu.

DOI:10.1523/JNEUROSCI.2349-04.2004

Copyright $\odot 2004$ Society for Neuroscience $\quad$ 0270-6474/04/248428-08\$15.00/0 studies suggest that binding capability in vitro does not necessarily reveal ligand-receptor function in vivo.

In vivo, Sema3A is believed to act through Npn-1 for its repulsive activity, based on the similar phenotypes observed in $n p n-1$ and sema3A knock-out mice (Kitsukawa et al., 1997; Taniguchi et al., 1997). Likewise, Npn-2 appears to mediate Sema3F-induced repulsion in vivo (Chedotal et al., 1998; Giger et al., 1998; Sahay et al., 2003). Explant and growth cone collapse assays suggest that Sema3C functions through Npn-1-Npn-2 heterodimers (Chen et al., 1998; Takahashi et al., 1998); however, whether an individual semaphorin acts via different neuropilin complexes to elicit different axonal responses is unknown.

Here, we demonstrate that Sema3D is required for correct formation of the early axon pathways in the zebrafish brain and that different functions of Sema3D may require different neuropilin components. In zebrafish, there are four neuropilins, Npn-1A, Npn1B, Npn-2A, and Npn-2B (Lee et al., 2002; Bovenkamp et al., 2004; Yu et al., 2004). Our results indicate that Sema3D repels axons of the nucleus of the medial longitudinal fasciculus (nucMLF) and is required for their subsequent fasciculation. Proper repulsion and fasciculation of these axons also requires Npn-1A. Results from Sema3D knock-down and ectopic misexpression experiments suggest that Sema3D attracts telencephalic axons to the midline to form the anterior commissure (AC). Formation of the $\mathrm{AC}$ also requires both Npn-1A and Npn-2B. These results, together with combined partial knock-down experiments, provide evidence that axons may respond differentially to a single semaphorin, depending on their neuropilin composition.

\section{Materials and Methods}

Animals. Zebrafish (Danio rerio) were maintained in a laboratory breeding colony on a 14/10 hr light/dark cycle. Collected embryos were main- 
tained at $28.5^{\circ} \mathrm{C}$ and staged as described previously (Kimmel et al., 1995). Embryo stage was defined as hours post-fertilization (hpf).

In situ hybridization and immunohistochemistry. Digoxygenin-UTPlabeled riboprobes for sema3D, $n p n-1 A, n p n-1 B, n p n-2 A$, and $n p n-2 B$ were synthesized by in vitro transcription and hydrolyzed to an average length of $\sim 300$ bases by limited alkaline hydrolysis (Cox et al., 1984). Whole-mount in situ hybridization was performed as described previously (Halloran et al., 1999).

For whole-mount immunohistochemistry, embryos were fixed in $4 \%$ paraformaldehyde overnight, blocked in $5 \%$ sheep serum and $2 \mathrm{mg} / \mathrm{ml}$ BSA in PBS, and incubated overnight $\left(4^{\circ} \mathrm{C}\right)$ in the monoclonal antibody ZN-12 (1:500; Zebrafish International Resource Center, Eugene, OR) or anti-acetylated $\alpha$-tubulin (1:1500; Sigma, St. Louis, MO). Antibody labeling was performed with the Vectastain Mouse IgG ABC immunoperoxidase labeling kit (Vector Laboratories, Burlingame, CA). For fluorescent double labeling, embryos were fixed and blocked as described above. Embryos were then incubated simultaneously overnight $\left(4^{\circ} \mathrm{C}\right)$ in antiacetylated $\alpha$-tubulin (1:1500; Sigma) and rabbit-anti-green fluorescent protein (GFP) (0.4 $\mu \mathrm{g} / \mathrm{ml}$; Sigma). Alexa 488 - and Alexa 568-conjugated secondary antibodies ( $4 \mu \mathrm{g} / \mathrm{ml}$; Molecular Probes, Eugene, OR) were used to bind anti-tubulin and anti-GFP, respectively.

Mosaic Sema3D misexpression. We used the zebrafish $h s p 70$ promoter (Halloran et al., 2000) to drive ectopic expression of sema3D. Embryos were injected into the blastomere at the one-cell stage with $\sim 1 \mathrm{nl}$ of a 50 $\mu \mathrm{g} / \mathrm{ml}$ solution of $h s p: s e m a 3 D-g f p$ or $h s p: g f p$ DNA. DNA constructs were prepared with a Qiagen (Hilden, Germany) Maxi-prep kit. To induce the hsp70 promoter and gene expression, embryos were placed in a $39^{\circ} \mathrm{C}$ water bath for $1 \mathrm{hr}$.

Morpholino antisense. Morpholino oligonucleotides against sema3D, $n p n-1 A, n p n-2 A$, and $n p n-2 B$ were synthesized by Gene Tools (Corvallis, $\mathrm{OR})$. The sequence of the sema3D blocking morpholino (3DMO) is as follows (with the sequence complementary to the start codon underlined): 5 '-CATGATGGACGAGGAGATTTCTGCA-3'. The control morpholino (CONMO) consisted of the identical sequence with four mispaired bases (lowercase): 5' -CATcATGcACGAGGAGATaTCTcCA$3^{\prime}$. The sequences of the $n p n-1 A$ (1AMO), $n p n-2 A$ (2AMO), and $n p n-2 B$ (2BMO) blocking morpholinos are as follows (sequence complementary to the start codon underlined or residue numbers included for sequences not overlapping with start codon): 1AMO, (-5) 5'-GAATCCTGGAGTTCGGAGTGCG-GAA-3' (-30); 2AMO, 5'-CTTGGTGTGATATCCAGAAATCCAT-3'; 2BMO, (-28) 5' -CGCGTAGAGGAAAAAGCTGAAGTTC- $3^{\prime}(-52)$. For the neuropilin morpholinos, a random sequence was used for a control (STDCON): $5^{\prime}$-CCTCTTACCTCAGTTACA-ATTTATA-3'. To test for morpholino specificity, we performed BLAST (basic local alignment search tool) analysis of the morpholino sequences against the Sanger Institute's zebrafish genome sequence assembly. None of the morpholinos are predicted to bind to gene sequences other than those targeted.

Morpholino oligos were injected into newly fertilized embryos at the $1-4$ cell stage as described previously (Nasevicius and Ekker, 2000). The following dose ranges of each morpholino were used in this paper to elicit full knock-down: 3DMO $(100-500 \mu \mathrm{M}), 1 \mathrm{AMO}(250 \mu \mathrm{M})$, and 2BMO $(500 \mu \mathrm{M})$. Equivalent doses of appropriate controls were injected for each experiment. Subthreshold concentrations used in combination to produce MLF defects were $25 \mu \mathrm{M} 3 \mathrm{DMO}$ plus $50 \mu \mathrm{M}$ 1AMO. The control single subthreshold doses were $25 \mu \mathrm{M}$ 3DMO plus $50 \mu \mathrm{M}$ STDCON, or 50 $\mu \mathrm{M} 1 \mathrm{AMO}$ plus $25 \mu \mathrm{M}$ STDCON (abbreviated in Fig. 6 as SUBCON). Concentrations used to cause AC errors were $50 \mu \mathrm{M} 3 \mathrm{DMO}$ plus $100 \mu \mathrm{M}$ $1 \mathrm{AMO}$ or $250 \mu \mathrm{M} 2 \mathrm{BMO}$. In all cases, control single subthreshold doses were the same concentration of the particular translation-blocking morpholino combined with the appropriate dose of STDCON.

In vitro transcription-translation. Coupled in vitro transcriptiontranslation (TnT Coupled Reticulocyte Lysate Systems, Promega, Madison, WI) was performed on $n p n-1 A, n p n-2 A$, and $n p n-2 B$ DNA inserted into the pcDNA3.1 ${ }^{+}$vector (Invitrogen, Gaithersburg, $\mathrm{MD}$ ). Incorporation of biotinylated lysine residues (Transcend tRNA, Promega) during translation allowed for detection of protein products. Translation was performed on each DNA type alone ( $1 \mu \mathrm{g}$; Qiagen Maxi-prep) and in the presence of $10 \mu \mathrm{M}$ STDCON or $10 \mu \mathrm{M}$ of the appropriate translation- blocking morpholino. Protein products were separated by SDS-PAGE (4-20\% Express Mini Gel, Gene Mate) and transferred to polyvinylidene difluoride membrane using a semidry transfer apparatus (Bio-Rad, Hercules, CA). The membrane was incubated in streptavidin-HRP (1:5000; Promega), and protein products were detected via chemiluminescence.

Imaging. All bright-field images were captured on a Nikon TE300 inverted microscope equipped with a Spot RT camera (Diagnostic Instruments, Sterling Heights, MI) and processed with MetaMorph software (Universal Imaging, West Chester, PA). Fluorescent images are confocal projections captured on a Zeiss Axiovert 100M microscope and the Bio-Rad 1024 Lasersharp Confocal. Step sizes were $1 \mu \mathrm{m}$.

\section{Results}

\section{Expression of sema3D and neuropilins}

We previously reported the spatial-temporal expression pattern of sema3D in the developing zebrafish brain (Halloran et al., 1999). The relationship of sema3D expression to developing axon pathways suggests that it may play a role in their guidance. Specifically, sema3D is expressed in the neuroepithelium of the rostral ventral midbrain, extending into the ventral diencephalon (Fig. 1A). Immediately caudal to this domain are the bilateral nuclei of the nucMLF (Fig. $1 B$ ). Each nucMLF extends axons caudally, away from the sema3D-expressing cells, forming the two MLFs that extend on either side of the ventral midline. sema3D is also expressed in the midbrain floor plate, medial to these two axon tracts, when the axons are first extending (Fig. 1 $\mathrm{A}$ ) (Halloran et al., 1999). In addition, sema3D is expressed in the telencephalon at the telencephalic-nasal placode border, which is dorsolateral to the telencephalic neurons that extend to form the AC (Fig. 1C,D). Also, sema3D is expressed the midline near the AC (Fig. 1C). Thus, Sema3D is in a position to influence the development of multiple axon pathways, including those of the nucMLF and the AC.

The initial tactic that we used to determine whether Sema3D might regulate pathfinding by neurons of the nucMLF or AC was to examine the expression of potential Sema3D receptor components within these neurons. Chick Sema3D has been shown to bind Npn-1, suggesting that it acts as part of the Sema3D receptor complex (Feiner et al., 1997); however, no function or activity for Sema3D in chick has been described. Therefore, it is not known whether Sema3D functions specifically through Npn-1. We examined the expression of the four zebrafish neuropilins in the region of the nucMLF and the telencephalon. $n p n-1 A$ is expressed in the ventral midbrain in the area that includes the cell bodies of the nucMLF (Fig. $1 E$ ) and in the telencephalic neuron clusters that form the AC (Fig. $1 F$ ). $n p n-2 B$ is also expressed in the telencephalic neurons (Fig. $1 H$ ), but not in the ventral midbrain. $n p n-2 A$ is expressed lateral to and overlapping with a subset of the telencephalic neurons (Fig. $1 G$ ), but it is not expressed in the ventral midbrain. $n p n-1 B$ was expressed by neither the nucMLF nor the telencephalic neurons (data not shown). Together, these expression patterns suggest that the nucMLF and telencephalic neurons are likely responsive to class 3 semaphorins.

\section{Sema3D knock-down disrupts axon pathway formation}

To investigate the role of Sema3D in guiding axon pathways, we used morpholino antisense to knock down Sema3D protein. We examined the effects of Sema3D knock-down by labeling axons with either the ZN-12 antibody, which labels a subset of early axon pathways (Metcalfe et al., 1990), or anti-tubulin, which labels all axon pathways at these stages (Chitnis and Kuwada, 1990). We have shown previously that a morpholino oligo designed against the translation start site of sema3D effectively blocks Sema3D protein expression (Liu et al., 2004). Embryos 

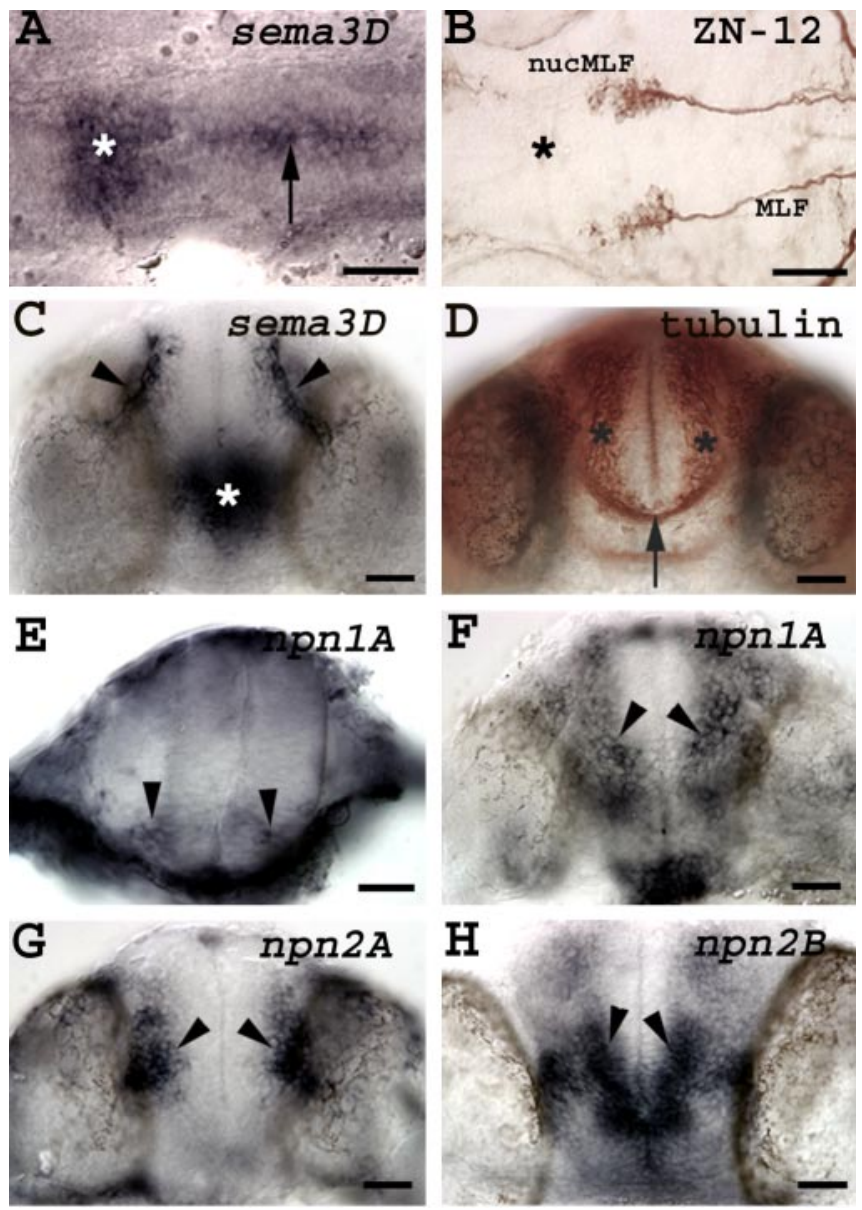

Figure 1. Expression of sema3D and neuropilin mRNAs in the developing zebrafish brain. $A$, $B$, Ventral views, anterior to the left, of diencephalon-midbrain region of $18 \mathrm{hpf}$ embryos. $A, I n$ situ hybridization for sema3D shows expression in the ventral diencephalon (asterisk) and the midbrain floor plate (arrow). B, Corresponding view of ZN-12-labeled embryo shows the bilateral nucMLFs, which lie caudal to the sema3D expression and extend axons caudally to form the MLF. Asterisk corresponds to location of asterisk in $A$ denoting sema3D expression. C, D, Rostral views of telencephalon region of $24 \mathrm{hpf}$ embryos. C, In situ hybridization for sema3D shows expression at the telencephalic-nasal placode border (arrowheads), dorsolateral to the telencephalic neurons, and in the midline region near the AC (asterisk). D, Corresponding view of an embryo labeled with anti-tubulin shows telencephalic neurons (asterisks) that extend axons to form the $\mathrm{AC}$ (arrow). E, Cross section of the midbrain shows npn-1A expression (arrowheads) in the region of the nucMLF. $F-H$, Rostral views of 24 hpf embryos showing npn- $1 A$ $(F), n p n-2 A(G)$, and $n p n-2 B(H)$ expression (arrowheads) by the telencephalic neurons. Scale bar, $50 \mu \mathrm{m}$.

were injected at the 1-4 cell stage with $3 \mathrm{DMO}$ or CONMO and allowed to develop to specific stages of axon outgrowth. The 3DMO-injected embryos displayed normal morphology throughout the earlier stages of development.

Based on the spatial-temporal relationship between sema3D expression and the nucMLF axon projection, we hypothesized that Sema3D acts to repel these axons caudally toward the hindbrain and spinal cord. Sema3D knock-down caused two types of guidance defects in nucMLF axon outgrowth. First, in $48 \%(n=$ 63) of 3DMO-injected embryos, we found that one to three nucMLF neurons extended their axons rostrally into the diencephalon rather than caudally (Fig. $2 B$, Table 1 ). This type of error occurred very rarely $(3 \% ; n=37)$ in CONMO-injected embryos (Fig. 2A, Table 1). These misprojected axons grow through the ventral midbrain region where Sema3D is normally expressed, suggesting that it acts to prevent nucMLF axons from extending in the rostral direction.
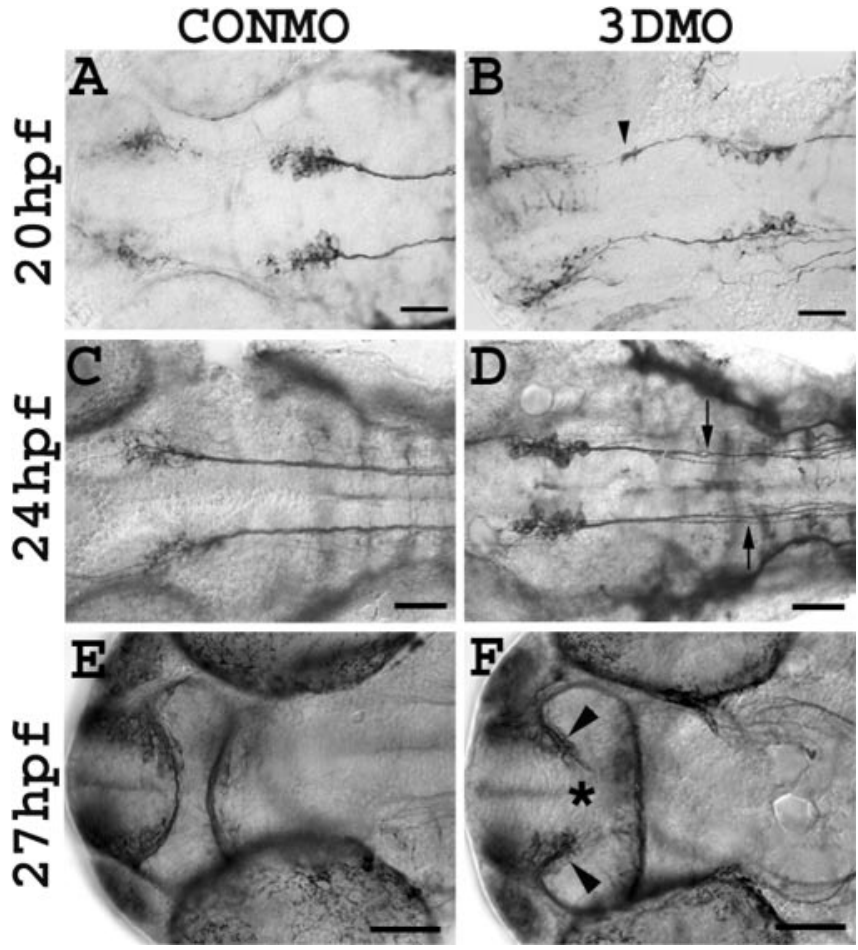

Figure 2. Knock-down of Sema3D affects axon path formation. All are ventral views, anterior to the left, of whole-mount embryos. $A, B, Z \mathrm{~N}-12$ labeling showing the nucMLF in $20 \mathrm{hpf}$ embryos injected with CONMO $(A)$ or 3DMO $(B)$. NucMLF axons extend rostrally after Sema3D knock-down ( $B$, arrowhead). $C, D, Z N-12$ labeling of $24 \mathrm{hpf}$ embryos injected with CONMO ( $C$ or 3DMO (D). The MLFs are defasciculated after Sema3D knock-down (D, arrows).E, $F$, Anti-tubulin labeling showing the $\mathrm{AC}$ in $27 \mathrm{hpf}$ embryos injected with CONMO $(E)$ or 3DMO ( $F$ ). The AC fails to form after Sema3D knock-down ( $F$, asterisk). Scale bar, $50 \mu \mathrm{m}$.

In addition to aberrant rostral projections, the nucMLF neurons showed another axon guidance defect after Sema3D knockdown. In $69 \%(n=139)$ of 3DMO-injected embryos, the axons that projected caudally to form the MLF were defasciculated in the rostral hindbrain (rhombomeres 1-3) (Fig. 2D, Table 1). Defasciculation was defined by observable gaps between axons of the MLF, causing an increase in width of the overall tract. The defasciculated axons were not biased medial or lateral to the fascicle; rather they were centered on the normal location of the fascicle. In most CONMO-injected embryos, the MLF axons form a tight fascicle (Fig. 2C, Table 1); however, we observed a low degree of defasciculation in $8 \%(n=145)$ of these controls. These results indicate that Sema3D is necessary for proper fasciculation of the MLF in the rostral hindbrain.

Next, we investigated the role of Sema3D in guiding axons of the telencephalic neurons. At 20-24 hpf, these neurons extend axons to form the AC, which connects the bilateral telencephalic neuronal clusters (Chitnis and Kuwada, 1990). Because sema3D is expressed by the cells dorsolateral to the telencephalic neurons and near the midline-spanning region of the AC during its formation, we hypothesized that Sema3D knock-down might alter the development of the AC. In fact, 63\% $(n=39)$ of 3DMOinjected embryos exhibited defects in the formation of the $\mathrm{AC}$ (Fig. 2F, Table 1). In these embryos, telencephalic neurons did not extend axons medially to form the AC, and in some cases the axons appeared to form swirls and bundles in close proximity to the clusters of telencephalic neurons (Fig. $2 F$ ). Failure of the AC to develop fully was observed in only $7 \%(n=30)$ of the control group (Fig. $2 E$, Table 1). These results indicate that Sema3D is required for proper formation of the AC. 
Table 1. Knock-down of Sema3D, Npn-1A, and Npn-2B causes axon guidance defects

\begin{tabular}{lccc}
\hline Injection type & $\begin{array}{l}\text { Embryos with rostral } \\
\text { nucMLF axons }(\%)\end{array}$ & $\begin{array}{l}\text { Embryos with } \\
\text { defasciculated MLFs }(\%)\end{array}$ & $\begin{array}{l}\text { Embryos with } \\
\text { unformed AC (\%) }\end{array}$ \\
\hline CONM0 & $3(n=37)$ & $8(n=145)$ & $7(n=30)$ \\
3DM0 & $48(n=63)^{*}$ & $69(n=139)^{*}$ & $63(n=39)^{*}$ \\
STDCON & $0(n=20)$ & $0(n=23)$ & $10(n=20)$ \\
1AM0 & $48(n=27)^{*}$ & $82(n=112)^{*}$ & $74(n=31)^{*}$ \\
2BM0 & & $50(n=28)^{* *}$ \\
\hline
\end{tabular}

Morpholino doses described in Materials and Methods. Two sample comparisons were made for each experimental grouping (i.e., 3DM0 vs (ONM0), and SDs were calculated assuming a binomial distribution with the percentages above and each sample size. ${ }^{*} p<0.001 ;{ }^{* *} p<0.01$.
GFP

tubulin

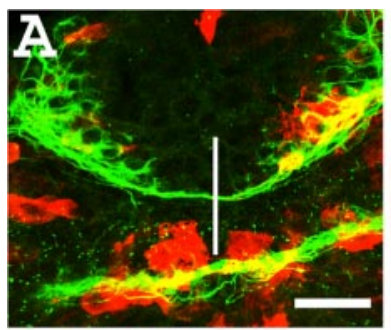

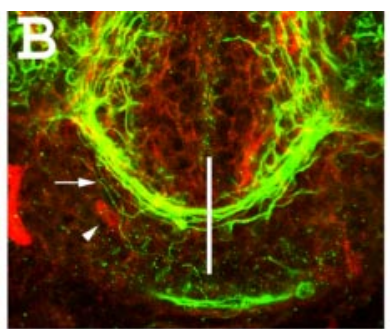

Sema3D-GFP

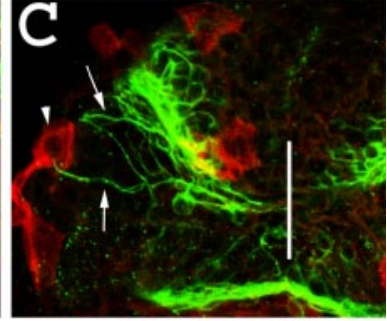

Sema3D-GFP
Figure 3. Sema3D mosaic misexpression attracts axons from the anterior commissure. $A-C$, Confocal projections of $A C$ region of embryos labeled with anti-tubulin (green) and anti-GFP (red). A, Embryo injected with hsp:gfp DNA. GFP-expressing cells do not attract axons from the AC. B, C, Embryos injected with hsp:sema3D-gfp DNA. Axons leave the pathway of the AC to extend toward ectopic Sema3D-expressing cells. Arrowheads indicate ectopic Sema3D expression, and arrows indicate aberrant axons. Vertical white line denotes midline. Scale bar, $50 \mu \mathrm{m}$.

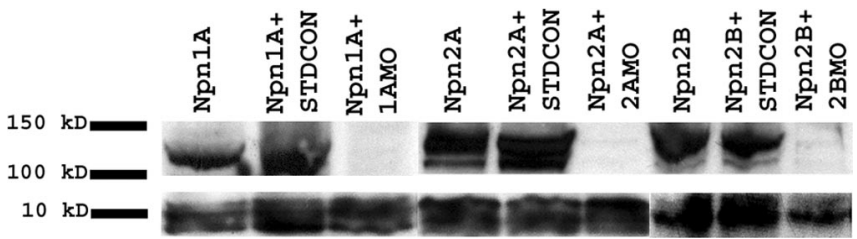

Figure 4. Morpholinos block translation of Npn-1A, $-2 A$, and $-2 B$ in vitro. Protein bands detected by chemiluminescence after in vitro translation of npn-1A, npn-2A, and npn-2B alone, in the presence of a control morpholino or in the presence of morpholinos designed to block translation of Npn-1A, Npn-2A, or Npn-2B. A 10 kDa band of an endogenously biotinylated reaction product is shown as a loading control for each lane.

Furthermore, we tested the specificity of these effects of Sema3D knock-down by knocking down the most closely related semaphorin, Sema3A1. sema3A1 is expressed in the region of the nucMLF and in the rostral diencephalon (Yee et al., 1999), where it could potentially affect the development of nucMLF and AC axons. We injected a morpholino sequence that has been shown previously to effectively block translation of Sema3A1 (Shoji et al., 2003). Knock-down of Sema3A1 failed to affect the extension direction of the nucMLF axons, fasciculation of the MLF, or the formation of the AC (data not shown). Thus, the guidance defects observed in Sema3D knock-down are not ubiquitous for all class 3 semaphorins and may be specific to Sema3D loss of function.

\section{Sema3D is attractive to telencephalic axons in vivo}

Our results from the Sema3D knock-down experiments are most consistent with an attractive role for Sema3D in guiding telencephalic axons. If Sema3D in the dorsolateral telencephalon acted to repel axons toward the midline, we might expect to see axons extending into this dorsolateral domain after Sema3D knockdown. We never observed dorsolateral axon extension in any of the 3DMO-injected embryos, suggesting that Sema3D does not act as a lateral repellant to drive telencephalic axons toward the midline. Instead, axons remained in close proximity to their cell bodies, well lateral to the midline.

To determine whether Sema3D is indeed attractive to these axons in vivo, we ectopically misexpressed Sema3D in a mosaic pattern. We injected a DNA construct encoding GFP-tagged Sema3D driven by the $h s p 70$ heat-inducible promoter (Halloran et al., 2000) (hsp:sema3D-gfp) into 1-cell stage embryos. We induced ectopic Sema3D-GFP expression by heating the embryos at stages before telencephalic axon outgrowth and fixed the embryos after the stage of AC formation. hsp:gfp constructs were injected into separate embryos as a control. The embryos were colabeled with anti-GFP to recognize the Sema3D-GFP and with anti-tubulin to label the axon pathways.

In $58 \%(n=19)$ of the hsp:sema3D$g f p$-injected embryos with cells expressing ectopic Sema3D-GFP in the vicinity of the AC, axons extending from the telencephalic neurons strayed away from the tract of the $\mathrm{AC}$ and grew toward the ectopic Sema3D-GFP (Fig. 3B,C). On average, two to five axons deviated from the commissure and extended toward ectopic Sema3D-expressing cells near the AC (Fig. $3 B, C$ ). One extreme case was observed in which an ectopic Sema3D cell located lateral to the telencephalic neurons caused axons to grow away from the commissure, possibly preventing commissure formation (Fig. $3 C$ ). All of the control embryos with GFP-expressing cells near the AC $(n=16)$ possessed an established AC in which the axons did not exit the tract and extend toward the GFP-expressing cells (Fig. 3A). These results support the hypothesis that Sema3D in the midline region attracts the axons extending from the telencephalic neurons to form the AC.

\section{Neuropilin-1A and neuropilin-2B are necessary for the} development of nucMLF and $\mathrm{AC}$ axon pathways in vivo

To gain insight into the specific receptor components that mediate the effects of Sema3D, we used morpholino antisense to individually knock down each of the zebrafish neuropilins expressed in the nucMLF or telencephalon. As a control we used a random nonspecific 25-mer oligo, STDCON. We tested the efficacy of our morpholinos with an in vitro translation assay. This assay verified that the morpholinos designed to target $n p n-1 A, n p n-2 A$, and $n p n-2 B$ effectively blocked translation (Fig. 4 ), whereas the STDCON did not block or reduce translation. Embryos injected with the neuropilin-blocking morpholinos did not show morphological defects during stages of development before axon outgrowth.

Knock-down of a critical receptor component for Sema3D should phenocopy the effects of Sema3D knock-down. We found that injection of morpholinos against Npn-1A (1AMO) caused the same defects in nucMLF and telencephalic axon pathfinding observed with Sema3D knock-down. In 48\% ( $n=27)$ of 1AMOinjected embryos, we found axons extending rostrally from the nucMLF (Fig. 5B, Table 1). None of the STDCON-injected embryos $(n=20)$ displayed this phenotype (Fig. 5A, Table 1). Furthermore, $82 \%(n=112)$ of 1AMO-injected embryos exhibited 


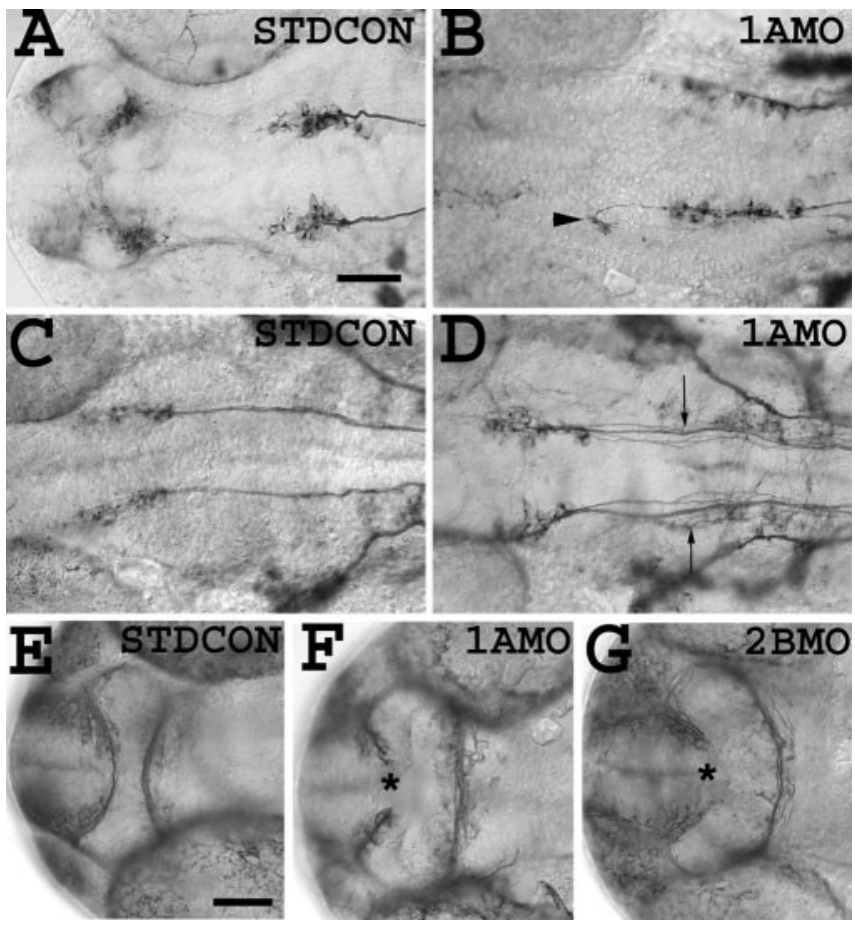

Figure 5. Neuropilin knock-down phenocopies Sema3D knock-down. All are ventral views, anterior to the left, of whole-mount embryos. $A, B, Z \mathrm{~N}-12$ labeling showing the nucMLF in 20 hpf embryos injected with STDCON $(A)$ or 1AMO $(B)$. NucMLF axons extend rostrally after Npn-1A knock-down (arrowhead). C, D, ZN-12 labeling of $24 \mathrm{hpf}$ embryos injected with CONMO ( $C$ or 1 AMO (D). The MLF is highly defasciculated after Npn-1A knock-down (arrows). E-G, Anti-tubulin labeling showing the AC in 27 hpf embryos injected with $\operatorname{STDCON}(E), 1 \mathrm{AMO}(F)$, or 2BMO ( $G$ ). The AC fails to form after knock-down of Npn-1A or Npn-2B ( $F, G$, asterisks). Scale bar, $50 \mu \mathrm{m}$

marked defasciculation of the MLF in rhombomeres 1-3 (Fig. $5 D$, Table 1$)$ as compared with $0 \%$ of controls $(n=23)$ (Fig. $5 C$, Table 1). Not only did the incidence of MLF defasciculation increase after Npn-1A knock-down as compared with Sema3D knock-down, but MLF axons in 1AMO-injected embryos were qualitatively more dramatically defasciculated, spreading over a broader area. Knock-down of the other neuropilins had no effect on nucMLF outgrowth or fasciculation (data not shown). Finally, Npn-1A knock-down also prevented the formation of the AC in $74 \%$ of embryos $(n=31)$ (Fig. $5 F$, Table 1$)$, whereas $10 \%(n=$ 20) of STDCON-injected embryos had an unformed AC (Fig. 5E, Table 1). These results suggest that like Sema3D, Npn-1A is also required for proper guidance of nucMLF and AC axons.

Interestingly, we also observed failure of $\mathrm{AC}$ formation after knock-down of Npn-2B. The AC failed to form in $50 \%(n=28)$ of embryos injected with a morpholino against Npn-2B (2BMO) (Fig. $5 G$, Table 1$)$ versus $10 \%(n=20)$ of STDCON-injected embryos (Fig. 5E, Table 1). This phenotype was indistinguishable from that of Sema3D or Npn-1A knock-down in which the telencephalic axons formed bundles that did not cross the midline. Despite $n p n-2 A$ expression in a subset of telencephalic neurons, knock-down of Npn-2A did not disrupt AC formation (data not shown). Thus, Npn-2B, in addition to Sema3D and Npn-1A, appears to be required for axon growth through the AC.

\section{Genetic interactions among Sema3D, Npn-1A, and Npn-2B}

To further investigate which neuropilins might compose the Sema3D receptor, we investigated potential interactions among Sema3D, Npn-1A, and Npn-2B. We performed coinjection ex-
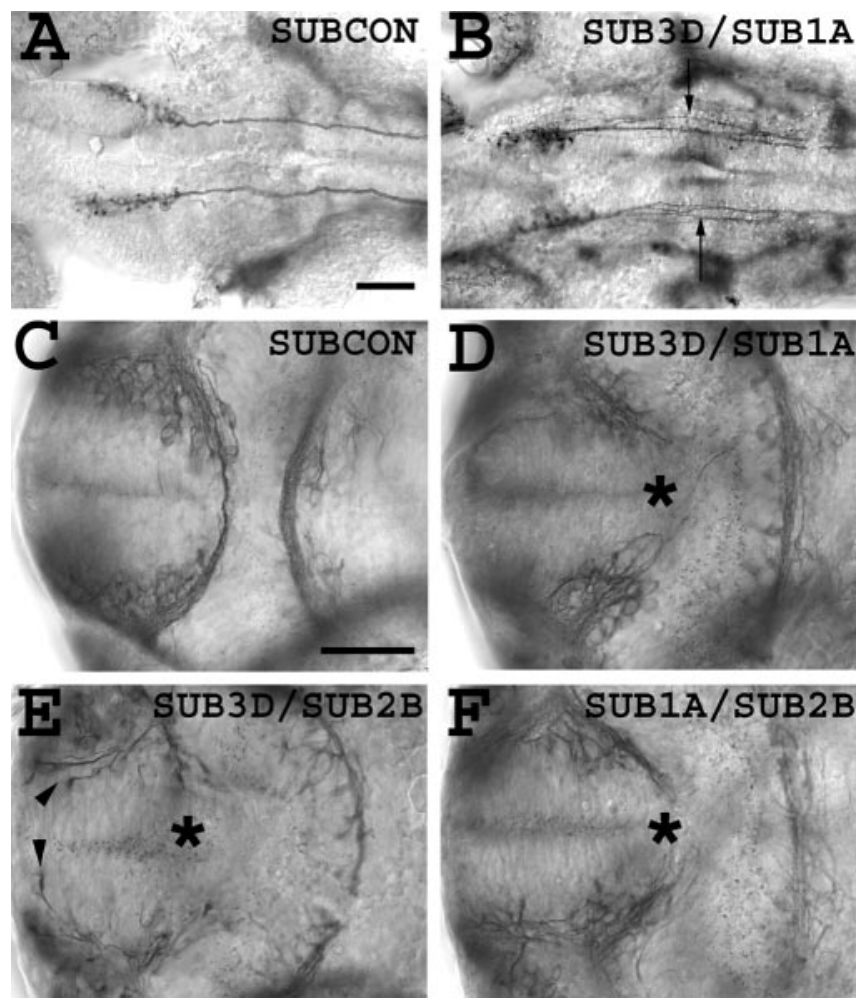

Figure 6. Simultaneous partial knock-down of Sema3D and neuropilins shows genetic interaction in vivo. All are ventral views, anterior to the left, of whole-mount embryos. $A, B, Z \mathrm{~N}-12$ labeling of $24 \mathrm{hpf}$ embryos injected with SUBCON $(A)$ or SUB3D-SUB1A $(B)$. This SUBCON embryo was injected with a subthreshold dose of 3DMO mixed with STDCON MO. Embryos injected with a subthreshold dose of 1 AMO plus STDCON had the same appearance. $B$, Arrows indicate defasciculation of the MLF in SUB3D-SUB1A-injected embryos. C $F$, Anti-tubulin labeling of $27 \mathrm{hpf}$ embryos injected with SUBCON (C), SUB3D-SUB1A (D), SUB3D-SUB2B $(E)$, and SUB1A-SUB2B $(F)$. Asterisks denote failure of $A C$ formation in embryos injected with combinations of subthreshold morpholino doses to sema3D, npn-1A, and npn-2B. Scale bar, $50 \mu \mathrm{m}$.

periments with subthreshold doses of morpholinos, in which we mixed low doses of two different morpholinos. We predicted that if Sema3D were acting through a particular neuropilin, then partial knock-down of the neuropilin would enhance the very weak phenotype caused by partial knock-down of Sema3D. This approach was designed to mimic double heterozygote studies in which a genetic interaction would suggest a functional ligandreceptor pair. As controls, we injected single subthreshold doses of each morpholino with enough standard control morpholino to make the final concentration equal to that injected in the coinjections (SUBCON; see Materials and Methods for concentrations). These subthreshold doses of each morpholino alone (referred to as SUB3D for Sema3D, SUB1A for Npn-1A, and SUB2B for Npn-2B) cause a partial knock-down of the protein but do not produce the robust phenotypic effects observed with a full dose of morpholino; however, simultaneous partial knock-down combinations of Sema3D, Npn-1A, and Npn-2B caused a strong increase in phenotypic effects.

Combined partial knock-down of Sema3D and Npn-1A caused defasciculation of the MLF and failure of the AC to develop. In $47 \%(n=34)$ of SUB3D-SUB1A-coinjected embryos, we observed a high degree of defasciculation (Fig. 6B, Table 2) compared with the tightly formed fascicles observed in all embryos injected with a single subthreshold morpholino $(n=43)$ (Fig. 6A, Table 2). In addition, simultaneous partial knock-down of Sema3D and Npn-1A blocked formation of the AC in 55\% 
Table 2. Partial knock-down combinations of Sema3D, Npn-1A, and Npn-2B affect axon path formation

\begin{tabular}{lcc}
\hline Injection type & $\begin{array}{l}\text { Embryos with } \\
\text { defasciculated MLFs (\%) }\end{array}$ & $\begin{array}{l}\text { Embryos with } \\
\text { unformed AC (\%) }\end{array}$ \\
\hline SUB3D-STDCON & $0(n=22)$ & $21(n=14)$ \\
SUB1A-STDCON & $0(n=21)$ & $23(n=13)$ \\
SUB3D-SUB1A & $47(n=34)^{*}$ & $55(n=22)^{* *}$ \\
SUB3D-STDCON & & $13(n=15)$ \\
SUB2B-STDCON & & $20(n=15)$ \\
SUB3D-SUB2B & & $46(n=28)^{* *}$ \\
SUB1A-STDCON & & $20(n=20)$ \\
SUB2B-STDCON & $9(n=22)$ \\
SUB1A-SUB2B & & $34(n=35)^{* *}$ \\
\hline
\end{tabular}

In each experimental grouping (see Materials and Methods for morpholino doses), control groups were not significantly different from each other and therefore were combined for two sample binomial comparisons with double partial knock-down groups. ${ }^{*} p<0.001 ;{ }^{* *} p<0.05$.

$(n=22)$ of the injected embryos (Fig. $6 D$, Table 2) compared with $21 \%$ of control embryos injected with SUB3D alone $(n=$ 14) (Fig. 6C, Table 2) and 23\% of those injected with SUB1A alone $(n=13)$ (Fig. $6 C$, Table 2). SUB3D-SUB1A-coinjected embryos, however, did not exhibit rostral extension errors by nucMLF axons, suggesting that the smaller amounts of endogenous Sema3D and Npn-1A remaining after partial knock-down may be sufficient to properly direct these axons caudally.

Simultaneous partial knock-down of Sema3D and Npn-2B also caused an increase in the incidence of AC defects. The AC did not form in $46 \%(n=28)$ of embryos injected with SUB3DSUB2B (Fig. 6E, Table 2). Only 13\% $(n=15)$ of embryos injected with SUB3D alone and 20\% $(n=15)$ of those injected with SUB2B alone (Fig. 6C, Table 2) showed AC defects. We also tested whether there was a genetic interaction between Npn-1A and Npn-2B, which would suggest that both neuropilins worked together to promote AC guidance. The AC failed to develop in 34\% $(n=35)$ of SUB1A-SUB2B-coinjected embryos (Fig. 6F, Table $2)$. In contrast, only $20 \%(n=20)$ of SUB1A alone- and $9 \%(n=$ 22) of SUB2B alone-injected embryos exhibited an unformed AC (Fig. 6C, Table 2). In each simultaneous partial knock-down experiment, the phenotype observed was indistinguishable from the previously described full knock-down defects of Sema3D, Npn-1A, and Npn-2B.

\section{Discussion}

In this study, we define three guidance roles for Sema3D in the formation of early axon tracts in the zebrafish brain. Sema3D knock-down indicates that Sema3D is necessary for guidance of nucMLF axons. First, it contributes to the initial decision of nucMLF axons to extend caudally. Sema3D is expressed rostral to the nucMLF, and Sema3D knock-down causes some nucMLF axons to extend aberrantly in the rostral direction, where Sema3D would normally be expressed. This result provides strong evidence that Sema3D is repulsive to these axons and is consistent with our previous results showing that Sema3D repels retinal ganglion cell axons (Liu et al., 2004). Knock-down of Npn-1A phenocopied Sema3D knock-down, suggesting that Npn-1A is a required component of the Sema3D receptor. Despite this result, simultaneous partial knock-down of Sema3D and Npn-1A did not produce rostral extension of nucMLF axons, which could argue against Sema3D and Npn-1A acting as a ligand-receptor pair. Knock-down of the other neuropilins did not affect the extension direction of nucMLF axons, however, indicating that they are not required and are likely not serving as the Sema3D receptor. Moreover, if Sema3D repels axons through
Npn-1A, it would follow the precedent set by the most closely related semaphorin, Sema3A, which requires Npn-1 for its repulsive actions in vitro and in vivo (He and Tessier-Lavigne, 1997; Kitsukawa et al., 1997; Kolodkin et al., 1997; Taniguchi et al., 1997). The apparent lack of interaction between Sema3D and Npn-1A could indicate that complete suppression of Sema3D and/or Npn-1A is required to produce misguided nucMLF axons.

Even after full knock-down of Sema3D or Npn-1A, the majority of nucMLF axons extended caudally. The incomplete penetrance of this phenotype suggests that these axons are also guided by other cues. Redundancy in guidance signals might be expected, given the importance of this initial directional decision. Perhaps there are other repulsive cues in the region of Sema3D expression and/or strong attractive signals caudal to the nucMLF. In fact, in most known cases, one axon decision point is regulated by multiple guidance signals. Perhaps the best studied case is the midline crossing decision of spinal commissural axons. These axons are attracted to the midline by netrin and sonic hedgehog (Serafini et al., 1996; Charron et al., 2003) and repelled from the midline by the combined activity of three slit genes (Long et al., 2004). Single mutations in any of these genes cause only some or no commissural axons to make midline errors (Serafini et al., 1996; Plump et al., 2002; Charron et al., 2003; Long et al., 2004). Alternatively, our Sema3D or Npn-1A full knock-down embryos may still have residual Sema3D-Npn-1A that is sufficient to repel most nucMLF axons. Although the morpholinos appeared to completely block translation in the in vitro assay, it is possible that small amounts of protein remain after knock-down in vivo.

Sema3D is also required for the fasciculation of nucMLF axons following their caudal extension. Fasciculation is important because late developing axons tend to follow fascicles set by earlier axons (Drazba and Lemmon, 1990) and because it contributes to correct innervation of targets by regulating where axons diverge from fascicles to invade target tissues (Tang et al., 1992, 1994; Rutishauser and Landmesser, 1996). Again, Npn-1A appears to be required for this effect of Sema3D, because Npn-1A knock-down mimics the defasciculation caused by Sema3D knock-down. Furthermore, we demonstrated a genetic interaction between Sema3D and Npn-1A in that the combined subthreshold morpholinos produced strong MLF defasciculation.

Class 3 semaphorins have been shown previously to be required for fasciculation. Both Sema3A and Npn-1 knock-out mice exhibit defasciculation of peripheral-cranial nerves (Kitsukawa et al., 1997; Taniguchi et al., 1997), and Sema3F and Npn-2 knock-out mice show defasciculation of several CNS pathways (Giger et al., 2000; Sahay et al., 2003); however, the mechanism by which semaphorins cause fasciculation is still not well defined. Both Sema3A and Sema3F are expressed in the tissues surrounding specific fascicles, suggesting that they mediate fasciculation by generating a repulsive surround. Sema3D is expressed in the midbrain floor plate, medial to the two MLFs, and could provide a generally inhibitory region around MLF axons. Sema3D knock-down does not cause MLF axons to converge on or cross the midline, however, as might be expected by the loss of a midline repellant. Rather, the defasciculation occurring after Sema3D or Npn-1A knock-down is centered on the normal location of the fascicles. Moreover, ectopic Sema3D-expressing cells lying lateral to the MLF did not cause these axons to divert their trajectory (M. A. Wolman and M. C. Halloran, unpublished observations). Together, these results suggest that Sema3D may not be mediating fasciculation through repulsion. Alternatively, Sema3D might function via a different mechanism to regulate the 
activity of adhesion molecules that mediate fasciculation. Evidence for the ability of class 3 semaphorins to regulate adhesion comes from studies showing that Sema3A directly modulates integrin activity and the adhesion of vascular endothelial cells (Serini et al., 2003). Neuropilins, in addition to forming part of the semaphorin receptor complex, can act as cell adhesion molecules (CAMs) to mediate axon fasciculation (Fujisawa et al., 1997) and cell aggregation, and aggregation is not caused by a direct interaction of semaphorins binding Npn-1 (Shimizu et al., 2000). Moreover, in some cases, the CAM L1 is a necessary component for the Sema3A receptor (Castellani et al., 2000, 2002, 2004). These dual functions for CAMs raise the possibility that there may exist complex cross-talk between semaphorins and CAMs, and perhaps semaphorin binding to neuropilin may modulate the adhesive properties of neuropilin itself or coreceptor components.

In addition to its roles in repulsion and fasciculation, Sema3D also may function as an attractive cue to the telencephalic axons that form the AC, possibly via a receptor containing Npn-1A and Npn-2B. Sema3D is expressed in the midline region near the AC, and after full or simultaneous partial knock-down of Sema3D, Npn-1A, and/or Npn-2B, axons fail to extend toward the midline. This phenotype is strikingly similar to the loss of the $\mathrm{AC}$ in mutant mice lacking the midline attractant netrin that also display bundling of AC axons lateral to the midline (Serafini et al., 1996); however, possible models of Sema3D function other than direct attraction cannot be ruled out. For example, Sema3D could antagonize binding of another repulsive semaphorin expressed at the midline, thus allowing AC axons to cross the midline. Similarly, Sema3B and Sema3C antagonize Sema3A binding to Npn-1 in vitro (Takahashi et al., 1998); however, our result showing that axons veered off the pathway of the AC toward ectopic Sema3D-expressing cells is more consistent with a model of direct attraction. Interestingly, Sema3F and Npn-2 act as a ligand-receptor pair to guide AC axons in mice (Giger et al., 2000; Sahay et al., 2003). It appears that Sema3F in surrounding neurons acts as a repellant to funnel axons into the AC. In Sema3F null mice, in conditional mutants lacking Sema3F only in neurons, or in Npn-2 knock-out mice, AC axons are misdirected or decussate in a highly disorganized manner (Sahay et al., 2003). On the contrary, in our knock-down phenotype, axons bundled lateral to the midline. It is interesting to speculate whether Npn-2 may interpret both repulsive and attractive cues to guide AC axons.

The fact that both Npn-1A and Npn-2B are required for AC formation and show genetic interaction in partial knock-down experiments with Sema3D and each other suggests that they may form a heterodimer in a functional, attractive Sema3D receptor. Similarly, it has been hypothesized that Sema3B and Sema3C may repel axons through Npn-1-Npn-2 heterodimers (Chen et al., 1998; Takahashi et al., 1998). Npn-1 and Npn-2 are capable of forming homodimers or heterodimers when coexpressed in COS cells in the absence of ligand (Chen et al., 1998). In addition, Sema3B and Sema3C each bind both Npn-1 or Npn-2 with equal affinities in vitro, and functional assays with neuronal explants suggest that both Npn-1 and Npn-2 may be required to mediate the repulsive activity of Sema3C (Chen et al., 1997; Takahashi et al., 1998). Our in vivo functional data provide evidence to support the idea that Sema3D-induced attraction is mediated by Npn-1A-Npn-2B heterodimers. Interestingly, both Sema3B and Sema3C, like Sema3D, have attractive as well as inhibitory activities: Sema3B for olfactory neurons (de Castro et al., 1999) and Sema3C for cortical neurons (Bagnard et al., 1998). It is not known which receptor components mediate attraction by Sema3B or Sema3C.

Our results show that a single semaphorin, Sema3D, has the potential to repel, attract, and modulate adhesion among axons in vivo. Moreover, our data support a model whereby Sema3D repels and regulates fasciculation via Npn-1A-containing receptors and attracts via Npn-1A-Npn-2B heterodimers. This model is reminiscent of netrin guidance, in which axons expressing DCC (deleted in colon cancer) are attracted to netrin; however, with coincident expression of UNC-5 (uncoordinated-5) they are repelled (Hong et al., 1999); however, no such mechanism has been demonstrated previously for semaphorins. For a complete understanding of semaphorin function, it will be crucial to also determine which particular neuropilins interact with specific plexins to confer specificity of growth cone response to semaphorin ligands.

\section{References}

Bagnard D, Lohrum M, Uziel D, Puschel AW, Bolz J (1998) Semaphorins act as attractive and repulsive guidance signals during the development of cortical projections. Development 125:5043-5053.

Bovenkamp DE, Goishi K, Bahary N, Davidson AJ, Zhou Y, Becker T, Becker CG, Zon LI, Klagsbrun M (2004) Expression and mapping of duplicate neuropilin-1 and neuropilin-2 genes in developing zebrafish. Gene Expr Patterns 4:361-370.

Castellani V, Chedotal A, Schachner M, Faivre-Sarrailh C, Rougon G (2000) Analysis of the L1-deficient mouse phenotype reveals cross-talk between Sema3A and L1 signaling pathways in axonal guidance. Neuron 27:237-249.

Castellani V, De Angelis E, Kenwrick S, Rougon G (2002) Cis and trans interactions of L1 with neuropilin-1 control axonal responses to semaphorin 3A. EMBO J 21:6348-6357.

Castellani V, Falk J, Rougon G (2004) Semaphorin3A-induced receptor endocytosis during axon guidance responses is mediated by L1 CAM. Mol Cell Neurosci 26:89-100.

Charron F, Stein E, Jeong J, McMahon AP, Tessier-Lavigne M (2003) The morphogen sonic hedgehog is an axonal chemoattractant that collaborates with netrin-1 in midline axon guidance. Cell 113:11-23.

Chedotal A, Del Rio JA, Ruiz M, He Z, Borrell V, de Castro F, Ezan F, Goodman CS, Tessier-Lavigne M, Sotelo C, Soriano E (1998) Semaphorins III and IV repel hippocampal axons via two distinct receptors. Development 125:4313-4323.

Chen H, Chedotal A, He Z, Goodman CS, Tessier-Lavigne M (1997) Neuropilin-2, a novel member of the neuropilin family, is a high affinity receptor for the semaphorins Sema E and Sema IV but not Sema III. Neuron 19:547-559.

Chen H, He Z, Bagri A, Tessier-Lavigne M (1998) Semaphorin-neuropilin interactions underlying sympathetic axon responses to class III semaphorins. Neuron 21:1283-1290.

Chitnis AB, Kuwada JY (1990) Axonogenesis in the brain of zebrafish embryos. J Neurosci 10:1892-1905.

Cox KH, DeLeon DV, Angerer LM, Angerer RC (1984) Detection of mRNAs in sea urchin embryos by in situ hybridization using asymmetric RNA probes. Dev Biol 101:485-502.

de Castro F, Hu L, Drabkin H, Sotelo C, Chedotal A (1999) Chemoattraction and chemorepulsion of olfactory bulb axons by different secreted semaphorins. J Neurosci 19:4437-4448.

Drazba J, Lemmon V (1990) The role of cell adhesion molecules in neurite outgrowth on Muller cells. Dev Biol 138:82-93.

Feiner L, Koppel AM, Kobayashi H, Raper JA (1997) Secreted chick semaphorins bind recombinant neuropilin with similar affinities but bind different subsets of neurons in situ. Neuron 19:539-545.

Fiore R, Puschel AW (2003) The function of semaphorins during nervous system development. Front Biosci 8:s484-499.

Fujisawa H (2004) Discovery of semaphorin receptors, neuropilin and plexin, and their functions in neural development. J Neurobiol 59:24-33.

Fujisawa H, Kitsukawa T, Kawakami A, Takagi S, Shimizu M, Hirata T (1997) Roles of a neuronal cell-surface molecule, neuropilin, in nerve fiber fasciculation and guidance. Cell Tissue Res 290:465-470.

Giger RJ, Urquhart ER, Gillespie SK, Levengood DV, Ginty DD, Kolodkin AL 
(1998) Neuropilin-2 is a receptor for semaphorin IV: insight into the structural basis of receptor function and specificity. Neuron 21:1079-1092.

Giger RJ, Cloutier JF, Sahay A, Prinjha RK, Levengood DV, Moore SE, Pickering S, Simmons D, Rastan S, Walsh FS, Kolodkin AL, Ginty DD, Geppert M (2000) Neuropilin-2 is required in vivo for selective axon guidance responses to secreted semaphorins. Neuron 25:29-41.

Halloran MC, Severance SM, Yee CS, Gemza DL, Raper JA, Kuwada JY (1999) Analysis of a zebrafish semaphorin reveals potential functions in vivo. Dev Dyn 214:13-25.

Halloran MC, Sato-Maeda M, Warren JT, Su F, Lele Z, Krone PH, Kuwada JY, Shoji W (2000) Laser-induced gene expression in specific cells of transgenic zebrafish. Development 127:1953-1960.

He Z, Tessier-Lavigne M (1997) Neuropilin is a receptor for the axonal chemorepellent Semaphorin III. Cell 90:739-751.

Hong K, Hinck L, Nishiyama M, Poo MM, Tessier-Lavigne M, Stein E (1999) A ligand-gated association between cytoplasmic domains of UNC5 and DCC family receptors converts netrin-induced growth cone attraction to repulsion. Cell 97:927-941.

Kimmel CB, Ballard WW, Kimmel SR, Ullmann B, Schilling TF (1995) Stages of embryonic development of the zebrafish. Dev Dyn 203:253-310.

Kitsukawa T, Shimizu M, Sanbo M, Hirata T, Taniguchi M, Bekku Y, Yagi T, Fujisawa H (1997) Neuropilin-semaphorin III/D-mediated chemorepulsive signals play a crucial role in peripheral nerve projection in mice. Neuron 19:995-1005.

Kolodkin AL, Ginty DD (1997) Steering clear of semaphorins: neuropilins sound the retreat. Neuron 19:1159-1162.

Kolodkin AL, Levengood DV, Rowe EG, Tai YT, Giger RJ, Ginty DD (1997) Neuropilin is a semaphorin III receptor. Cell 90:753-762.

Lee P, Goishi K, Davidson AJ, Mannix R, Zon L, Klagsbrun M (2002) Neuropilin-1 is required for vascular development and is a mediator of VEGF-dependent angiogenesis in zebrafish. Proc Natl Acad Sci USA 99:10470-10475.

Liu Y, Berndt J, Su F, Tawarayama H, Shoji W, Kuwada JY, Halloran MC (2004) Semaphorin3D guides retinal axons along the dorsoventral axis of the tectum. J Neurosci 24:310-318.

Long H, Sabatier C, Ma L, Plump A, Yuan W, Ornitz DM, Tamada A, Murakami F, Goodman CS, Tessier-Lavigne M (2004) Conserved roles for Slit and Robo proteins in midline commissural axon guidance. Neuron 42:213-223.

Metcalfe WK, Myers PZ, Trevarrow B, Bass MB, Kimmel CB (1990) Primary neurons that express the L2/HNK-1 carbohydrate during early development in the zebrafish. Development 110:491-504.

Nasevicius A, Ekker SC (2000) Effective targeted gene "knockdown" in zebrafish. Nat Genet 26:216-220.

Plump AS, Erskine L, Sabatier C, Brose K, Epstein CJ, Goodman CS, Mason
CA, Tessier-Lavigne M (2002) Slit1 and Slit2 cooperate to prevent premature midline crossing of retinal axons in the mouse visual system. Neuron 33:219-232.

Rutishauser U, Landmesser L (1996) Polysialic acid in the vertebrate nervous system: a promoter of plasticity in cell-cell interactions. Trends Neurosci 19:422-427.

Sahay A, Molliver ME, Ginty DD, Kolodkin AL (2003) Semaphorin 3F is critical for development of limbic system circuitry and is required in neurons for selective CNS axon guidance events. J Neurosci 23:6671-6680.

Serafini T, Colamarino SA, Leonardo ED, Wang H, Beddington R, Skarnes WC, Tessier-Lavigne M (1996) Netrin-1 is required for commissural axon guidance in the developing vertebrate nervous system. Cell 87:1001-1014.

Serini G, Valdembri D, Zanivan S, Morterra G, Burkhardt C, Caccavari F, Zammataro L, Primo L, Tamagnone L, Logan M, Tessier-Lavigne M, Taniguchi M, Puschel AW, Bussolino F (2003) Class 3 semaphorins control vascular morphogenesis by inhibiting integrin function. Nature 424:391-397.

Shimizu M, Murakami Y, Suto F, Fujisawa H (2000) Determination of cell adhesion sites of neuropilin-1. J Cell Biol 148:1283-1293.

Shoji W, Isogai S, Sato-Maeda M, Obinata M, Kuwada JY (2003) Semaphorin3a1 regulates angioblast migration and vascular development in zebrafish embryos. Development 130:3227-3236.

Takahashi T, Nakamura F, Jin Z, Kalb RG, Strittmatter SM (1998) Semaphorins $\mathrm{A}$ and $\mathrm{E}$ act as antagonists of neuropilin-1 and agonists of neuropilin-2 receptors. Nat Neurosci 1:487-493.

Tamagnone L, Comoglio PM (2000) Signaling by semaphorin receptors: cell guidance and beyond. Trends Cell Biol 10:377-383.

Tang J, Landmesser L, Rutishauser U (1992) Polysialic acid influences specific pathfinding by avian motoneurons. Neuron 8:1031-1044.

Tang J, Rutishauser U, Landmesser L (1994) Polysialic acid regulates growth cone behavior during sorting of motor axons in the plexus region. Neuron 13:405-414.

Taniguchi M, Yuasa S, Fujisawa H, Naruse I, Saga S, Mishina M, Yagi T (1997) Disruption of semaphorin III/D gene causes severe abnormality in peripheral nerve projection. Neuron 19:519-530.

Xu X, Ng S, Wu ZL, Nguyen D, Homburger S, Seidel-Dugan C, Ebens A, Luo Y (1998) Human semaphorin K1 is glycosylphosphatidylinositol-linked and defines a new subfamily of viral-related semaphorins. J Biol Chem 273:22428-22434.

Yee CS, Chandrasekhar A, Halloran MC, Shoji W, Warren JT, Kuwada JY (1999) Molecular cloning, expression, and activity of zebrafish semaphorin Z1a. Brain Res Bull 48:581-593.

Yu HH, Houart C, Moens CB (2004) Cloning and embryonic expression of zebrafish neuropilin genes. Gene Expr Patterns 4:371-378. 